\title{
Simvastatin inhibits smoke-induced airway epithelial injury: implications for COPD therapy
}

\author{
Benjamin B. Davis ${ }^{1}$, Amir A. Zeki ${ }^{2,3}$, Jennifer M. Bratt², Lei Wang ${ }^{1}$, \\ Simone Filosto ${ }^{3}$, William F. Walby ${ }^{4}$, Nicholas J. Kenyon ${ }^{2,3}$, Tzipora Goldkorn ${ }^{3}$, \\ Edward S. Schelegle ${ }^{4}$ and Kent E. Pinkerton ${ }^{1}$
}

Affiliations: ${ }^{1}$ Center for Health and the Environment, University of California, Davis, CA, ${ }^{2}$ Center for Comparative Respiratory Biology \& Medicine (CCRBM), University of California, Davis, CA, ${ }^{3}$ Division of Pulmonary, Critical Care, and Sleep Medicine, University of California, Davis, CA, ${ }^{4}$ Anatomy, Physiology and Cell Biology, School of Veterinary Medicine, University of California, Davis, CA, USA.

Correspondence: B.B. Davis, University of California at Davis, One Shields Ave., Davis, California, 95616, USA. E-mail: bbdavisवucdavis.edu

ABSTRACT Chronic obstructive pulmonary disease (COPD) is the third leading cause of death. The statin drugs may have therapeutic potential in respiratory diseases such as COPD, but whether they prevent bronchial epithelial injury is unknown. We hypothesised that simvastatin attenuates acute tobacco smokeinduced neutrophilic lung inflammation and airway epithelial injury.

Spontaneously hypertensive rats were given simvastatin $\left(20 \mathrm{mg} \cdot \mathrm{kg}^{-1}\right.$ i.p. $)$ daily for either 7 days prior to tobacco smoke exposure and during 3 days of smoke exposure, or only during tobacco smoke exposure.

Pretreatment with simvastatin prior to and continued throughout smoke exposure reduced the total influx of leukocytes, neutrophils and macrophages into the lung and airways. Simvastatin attenuated tobacco smoke-induced cellular infiltration into lung parenchymal and airway subepithelial and interstitial spaces. 1 week of simvastatin pretreatment almost completely prevented smoke-induced denudation of the airway epithelial layer, while simvastatin given only concurrently with the smoke exposure had no effect.

Simvastatin may be a novel adjunctive therapy for smoke-induced lung diseases, such as COPD. Given the need for statin pretreatment there may be a critical process of conditioning that is necessary for statins' anti-inflammatory effects. Future work is needed to elucidate the mechanisms of this statin protective effect.

@ERSpublications

Simvastatin pretreatment reduced smoke-induced airway epithelial injury in rats: possible adjunctive therapies in COPD? http://ow.ly/kDav5

Received: March 092012 | Accepted after revision: Oct 082012 | First published online: Nov 222012

Support statement: Grant support for this research is from the CTSC K12 Award KL2 RR 024144, American Asthma Foundation 09-0269, Tobacco-Related Disease Research Program (TRDRP) 18KT-0037, and 18XT-0154.

Conflict of interest: Disclosures can be found alongside the online version of this article at www.erj.ersjournals.com Copyright @ERS 2013 


\section{Introduction}

Chronic obstructive pulmonary disease (COPD) is the third leading cause of death in the USA, and current treatments do not significantly alter the progression of the disease [1]. Approximately 20 million adults in the USA have COPD, with a mortality of 20 out of 100000 [2]. The most common cause of COPD in the general population is cigarette smoking $[2,3]$. Persistent inflammation is a key component of COPD pathogenesis. Thickened bronchiolar walls accompanied by an influx of inflammatory cells, including neutrophils, macrophages and lymphocytes, are characteristic of COPD [4-8].

Besides lowering cholesterol, the statin drugs ("statins") possess pleiotropic anti-inflammatory, immunomodulatory, antioxidant and anti-proliferative properties, with potential clinical applications beyond cardiovascular disease $[9,10]$. Due to these pleiotropic effects, the statins have been proposed as a potential novel treatment for inflammatory respiratory diseases, including bronchial asthma and COPD [11, 12]. Compelling reductions in COPD morbidity, mortality and improvements in lung function have been attributed to the use of statins [11,13-18]. Additionally, statins have been found to reduce neutrophil transendothelial migration in cell culture $[19,20]$ and neutrophilic inflammation and influx into the lung tissues of animals [19, 21-24].

The anti-inflammatory effects of statins are partly mediated via inhibition of the mevalonate pathway. Specifically, statins inhibit the enzyme 3-hydroxy-3-methyl-glutaryl-CoA (HMG-CoA) reductase, the ratelimiting step in cholesterol biosynthesis. The immediate product of HMG-CoA reductase is mevalonate, which is metabolised to nonsterol isoprenoids, farnesyl pyrophosphate and geranylgeranyl pyrophosphate, and cholesterol. These metabolites are necessary for the post-translational isoprenylation and subsequent activation of intracellular monomeric small G-proteins (Ras, Rho, Rac, Cdc42, etc.). Monomeric small Gproteins (or GTPases) control many important biological functions, including immune cell function, cytoskeletal dynamics, transmigration and cell proliferation $[9,10,25]$, and are thought to mediate at least some of the beneficial effects of statins.

Others have published the beneficial effects of simvastatin in attenuating smoke-induced emphysema, pulmonary hypertension, and small airway remodelling [26, 27]. However, no studies have evaluated whether systemic treatment with statins inhibits smoke-induced large airway or bronchial epithelial damage. This question is highly relevant to human smoke-related lung diseases, such as COPD. Therefore, in this study we hypothesised that systemic treatment with simvastatin can: 1) attenuate acute tobacco smoke-induced lung inflammation; and 2) inhibit airway epithelial injury. We evaluated whether the mevalonate pathway and lung Ras and Rho GTPase activation were affected by treatment with simvastatin. We utilised the spontaneously hypertensive rat model of COPD because of its robust neutrophilic inflammatory response to tobacco smoke exposure [28-34]. This model produces COPD-like pathologies, which include mucin hypersecretion, airway epithelial squamation and thickening, airspace enlargement and pulmonary function changes, after 4 weeks of exposure to tobacco smoke. We chose to test simvastatin's effect with an acute tobacco smoke exposure that induces lung inflammation to isolate simvastatin's preventative effects on leukocyte recruitment and airway epithelial injury from possible effects of promoting recovery. In addition, this tobacco smoke exposure protocol allowed us to separate simvastatin's preventative effects on leukocyte recruitment and airway epithelial injury from prevention of mucus accumulation, due to mucin hypersecretion and the loss of cilliated epithelial cells, which occurs with a longer exposure to tobacco smoke.

\section{Materials and methods \\ Animals}

12-week-old spontaneously hypertensive male rats were purchased from Charles River Laboratories (Portage, MI, USA). Upon arrival, all animals were housed in polycarbonate cages under a $12 \mathrm{~h}$ light-dark pattern with continuous access to food and water. Animals were acclimated to the new housing environment for 1 week before exposure to tobacco smoke began. All animals were handled according to the US Animal Welfare Acts, and all procedures were performed under the supervision of the University Animal Care and Use Committee (University of California, Davis, USA).

\section{Tobacco smoke exposure}

Groups of six spontaneously hypertensive rats were each exposed to filtered air or to tobacco smoke $(\sim 80$ $90 \mathrm{mg} \cdot \mathrm{m}^{-3}$ total suspended particulates (TSP)) for $6 \mathrm{~h} \cdot$ day $^{-1}$ for 3 days. Whole body exposure to cigarette smoke was carried out using a TE10 smoke exposure system [35] that combusts 3R4F research cigarettes (Tobacco and Health Research Institute, University of Kentucky, KY, USA) with a $35 \mathrm{~mL}$ puff volume of $2 \mathrm{~s}$ duration once each minute (Federal Trade Commission smoking standard [36]). In addition to TSP, exposure conditions were monitored daily for both nicotine and carbon monoxide concentrations. 


\section{Simvastatin delivery}

Simvastatin was purchased from Sigma (St. Louis, MO, USA). Simvastatin was made as a $4 \mathrm{mg} \cdot \mathrm{mL}^{-1}$ stock solution that was dissolved in $10 \%$ ethanol as the drug vehicle. The drug vehicle was made with $100 \mu$ of ethanol, $150 \mu \mathrm{l}$ of $0.1 \mathrm{~N} \mathrm{NaOH}$, and $750 \mu \mathrm{l}$ of PBS to make $1 \mathrm{~mL}$ of $10 \%$ ethanol. Hydrochloric acid $(0.1 \mathrm{~N})$ was added as necessary to obtain a solution $\mathrm{pH}$ of 7.0.

Rats were given $20 \mathrm{mg} \cdot \mathrm{kg}^{-1}$ simvastatin via i.p. injection daily for either 1) 7 days prior to smoke exposure and $30 \mathrm{~min}$ prior to daily tobacco smoke exposures, or 2) only $30 \mathrm{~min}$ prior to daily exposure to tobacco smoke.

We chose this dose based on previously published work, by us and others, which has shown this to be an effective nontoxic dose [24,37]. It is difficult to extrapolate what the equivalent dose in humans would be.

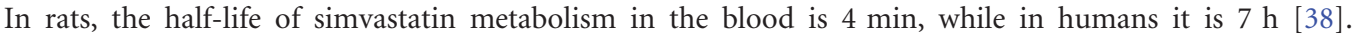
However, simvastatin is likely to remain at therapeutic levels in the tissues much longer.

\section{Pulmonary function test}

Rats were deeply anaesthetised with Ketamine and Xylazine 18-20 h following the final day of exposure to tobacco smoke. A midline incision was made over the cervical trachea. Once the rats were tracheocannulated, the cannula was then connected to the Scireq Flexivent (Scireq, Montreal, Canada) positivepressure ventilator, pulmonary-mechanics measurement and data acquisition system. Animals were paralysed by succinylcholine. The lung volume and pressure were measured twice while a standard respiratory cycle was simulated, once with the catheter open to room air and once with it closed. The rats were ventilated at a frequency of 90 breaths $\cdot \mathrm{min}^{-1}$ with a tidal volume of $10 \mathrm{~mL} \cdot \mathrm{kg}^{-1}$. Lung mechanics were evaluated using a forced oscillation technique. Measures of respiratory system input impedance were obtained that allow for the unique distinction between central and peripheral lung mechanics. A "snapshot perturbation" manoeuvre was imposed to measure resistance, compliance, and elastance of the whole respiratory system (airways, lung, and chest wall).

\section{Tissue preparation}

Following the pulmonary function test, rats were given an overdose of sodium pentobarbital. The trachea was cannulated, the left lung bronchus tied, and the right lung lavaged with $\mathrm{Ca}^{2+} / \mathrm{Mg}^{2+}$-free Hank's buffered salt solution (HBSS). Bronchoalveolar lavage (BAL) was performed using a three-in/three-out pattern of intratracheal instillation and removal with the same HBSS aliquot, in order to enrich total cell and protein recovery. BAL fluid (BALF) was collected in tubes and kept on ice prior to processing. The lavaged lung lobes were frozen in liquid nitrogen and stored at $-80^{\circ} \mathrm{C}$ until use. For histology, the suture on the left lung bronchus was released and the lung was inflated with $4 \%$ paraformaldehyde at $30 \mathrm{~cm}$ water pressure for $1 \mathrm{~h}$, followed by storage of the inflation-fixed lung immersed in fixative.

\section{Histology}

Histology was performed using cross-sectional lung tissue slices containing the first and second intrapulmonary airway generations from rats exposed to filtered air or tobacco smoke. Sections, $5 \mu \mathrm{m}$ thick, were cut from paraffin-embedded tissue blocks using a microtome. The sections were placed on glass slides and baked overnight at $37^{\circ} \mathrm{C}$ and subsequently deparaffinised in toluene and hydrated through a graded series of alcohol solutions. For haematoxylin and eosin staining, sections were stained with the following American MasterTech Scientific (Lodi, CA, USA) materials: Harris's haematoxylin, differentiating solution, bluing solution, and eosin Y stain. Sections were then dehydrated in ethanol and mounted with ClearMount (American MasterTech Scientific Inc.).

\section{BALF analysis}

The BALF was centrifuged at $250 \times g$ for $10 \mathrm{~min}$ at $4{ }^{\circ} \mathrm{C}$ to separate cells from the supernatant fluid. After centrifugation, the cell pellet was resuspended in $\mathrm{Ca}^{2+} / \mathrm{Mg}^{2+}$-free HBSS. The cell suspension was assayed for cell viability as determined by trypan blue exclusion. Total cell number was determined using a standard haemocytometer. Cytospin slides (Shandon, Pittsburgh, PA, USA) were prepared using aliquots of cell suspension that were then stained with Hema 3 (Fisher Scientific, Pittsburgh, PA, USA). Cell differentials in BALF were assessed by counting the number of macrophages, neutrophils, lymphocytes and eosinophils on the cytocentrifuge slides using light microscopy (500 cells counted per sample). The proportion of each cell type was multiplied by the total cell number per $\mathrm{mL}$ to determine total neutrophils, macrophages, lymphocytes and eosinophils per mL. Eosinophils are not reported since they made up $<0.01 \%$ of the total cells and were too few for statistical analysis. 


\section{Airway epithelial injury}

Airway epithelial injury was evaluated by measuring the percentage of sloughed airway epithelium. Using Image J software (National Institutes of Health, Bethesda, MD, USA) the length of sloughed airway epithelium was quantitatively measured and divided by the total airway epithelium in the haematoxylin and eosin stained lung sections at interpulmonary generation two. The p-values were determined using the Wilcoxon rank sum test.

\section{Western blot analysis of tissue}

Western blot methods are described in BRATT et al. [39] and were carried out with the following modifications. Sodium dodecyl sulphate polyacrylamide gel electrophoresis was performed using both cytosolic and membrane fractions (at $30 \mu \mathrm{g}$ total protein) under reducing conditions and transferred to a polyvinylidene difluoride membrane. Membranes were probed using $1 \mu \mathrm{g} \cdot \mathrm{mL}^{-1}$ rabbit anti-mouse Ras (Cell Signaling Technology Inc., Danvers, MA, USA); $1 \mu \mathrm{g} \cdot \mathrm{mL}^{-1}$ monoclonal anti-Rho (EMD Millipore, Billerica, MA, USA); $0.1 \mu \mathrm{g} \cdot \mathrm{mL}^{-1}$ monoclonal anti-E-cadherin (BD Bioscience, San Jose, CA, USA); $0.001 \mathrm{mg} \cdot \mathrm{mL}^{-1}$ anti-HO-1 (ABCam, Cambridge, MA, USA); or rabbit anti-mouse $\alpha$-Actinin IgG primary antibodies in $0.05 \%$ Tween in PBS (TBST), followed by incubation in $40 \mathrm{ng} \cdot \mathrm{mL}^{-1}$ horseradish peroxidase (HRP)conjugated goat anti-rabbit IgG (Pierce Biotechnology, Rockford, IL, USA) or HRP-conjugated goat antimouse IgG (R\&D Systems, Minneapolis, MN, USA) secondary antibodies in 5\% dry milk in TBST. Protein bands were then visualised using Western Lightning Plus-ECL substrate kit (PerkinElmer, Shelton, CT, USA) and Image Reader LAS-4000 V2.1 (Fuji Photo Film Co, Cypress, CA, USA). Individual band intensity was calculated using ImageJ (National Institutes of Health).

\section{Cholesterol assay}

Rat lungs were homogenised in a homogenisation buffer by hand using a dounce homogeniser as described above. Lung homogenate was centrifuged at $5000 \times \mathrm{g}$ for $5 \mathrm{~min}$ to remove large tissue debris. The supernatant was collected and aliquoted into volumes used to measure protein concentration and cholesterol. Lipids were isolated by adding a large volume of chloroform:methanol (2:1) solution, mixing (by vortex and sonication) and further addition of $1 \mathrm{M} \mathrm{NaCl}$ solution, which allows the separation of the organic phase (chloroform) from the aqueous phase by centrifugation $(3000 \times g$ for $5 \mathrm{~min})$ to occur. The bottom phase (chloroform) was collected and dried using a speed vacuum. Lipid pellets were resuspended in phosphate buffer $(0.1 \mathrm{M}$ potassium phosphate $\mathrm{pH} 7.4,50 \mathrm{mM} \mathrm{NaCl}, 5 \mathrm{mM}$ deoxycholic acid, $0.1 \%$ Triton X-100) and sonicated for at least $1 \mathrm{~min}$ to mix. Cholesterol was then measured via a fluorometric assay, the Amplex Red Cholesterol Assay Kit (Invitrogen, Grand Island, NY, USA), according to the manufacturer's instructions.

\section{Rho and Ras GTPase analysis}

Rat lung homogenates were prepared using isolation buffer (250 mM sucrose; $20 \mathrm{mM} \mathrm{HEPES,} \mathrm{pH} \mathrm{7.4;}$ $2 \mathrm{mM}$ EDTA; and $3 \mathrm{mM} \mathrm{NaN}_{3}$ ) containing protease inhibitors (protease inhibitor (Sigma, 1:100) and $1 \mathrm{mM}$ phenylmethylsulfonylfluoride) and phosphatase inhibitors (Phosphatase Inhibitor (Sigma, 1:100), $1 \mathrm{mM}$ $\mathrm{Na}_{3} \mathrm{VO}_{4}$, and $1 \mathrm{mM} \mathrm{NaF}$ ). Lung tissue homogenisation was performed by hand using a dounce homogeniser. Crude homogenates were initially centrifuged at $800 \times g$ for $5 \mathrm{~min}$ at $4^{\circ} \mathrm{C}$. The supernatant was then removed and saved as total lung homogenate. A $500 \mu \mathrm{L}$ aliquot of this total homogenate was centrifuged at $31000 \times g$ at $4{ }^{\circ} \mathrm{C}$ for $30 \mathrm{~min}$ to separate the cytosolic (soluble) and membrane (insoluble) components. The supernatant from this step was saved as the cytosolic fraction, and the remaining pellet was resuspended in $500 \mu \mathrm{L}$ of isolation buffer (same volume as the cytosolic fraction). The total protein concentration of the cytosolic fraction from each sample was measured using the Micro BCA Protein Assay Kit (Pierce Biotechnology) and was used to determine protein loading volumes for each sample.

We performed Western blots on our samples to assure that the cytosolic fractions were not contaminated by the membrane fractions and vice versa. We used E-cadherin as an indicator of membrane fractions since this protein is only expressed in cell membranes. None of our cytosolic fractions had evidence of membrane components and our membrane fractions positively confirmed E-cadherin expression. Previously, we also measured glyceraldehyde 3-phosphate dehydrogenase to confirm cytosolic fractions as further confirmation of our method, but these data are not included.

\section{Statistical analysis}

To increase the validity of tests of statistical significance, log-transformation was applied to outcome variables with skewed distributions before performing a one-way ANOVA with Bonferroni corrections for multiple comparisons where appropriate. The Wilcoxon rank sum test was used to analyse airway epithelial injury measurement and haem oxygenase (HO)-1 densitometric analysis due to grossly non-normal distributions. A p-value of $<0.05$ was used to indicate statistical significance. 
- Air $\square$ Air+simvastatin $\Delta$ Smoke $\nabla$ Smoke+simvastatin
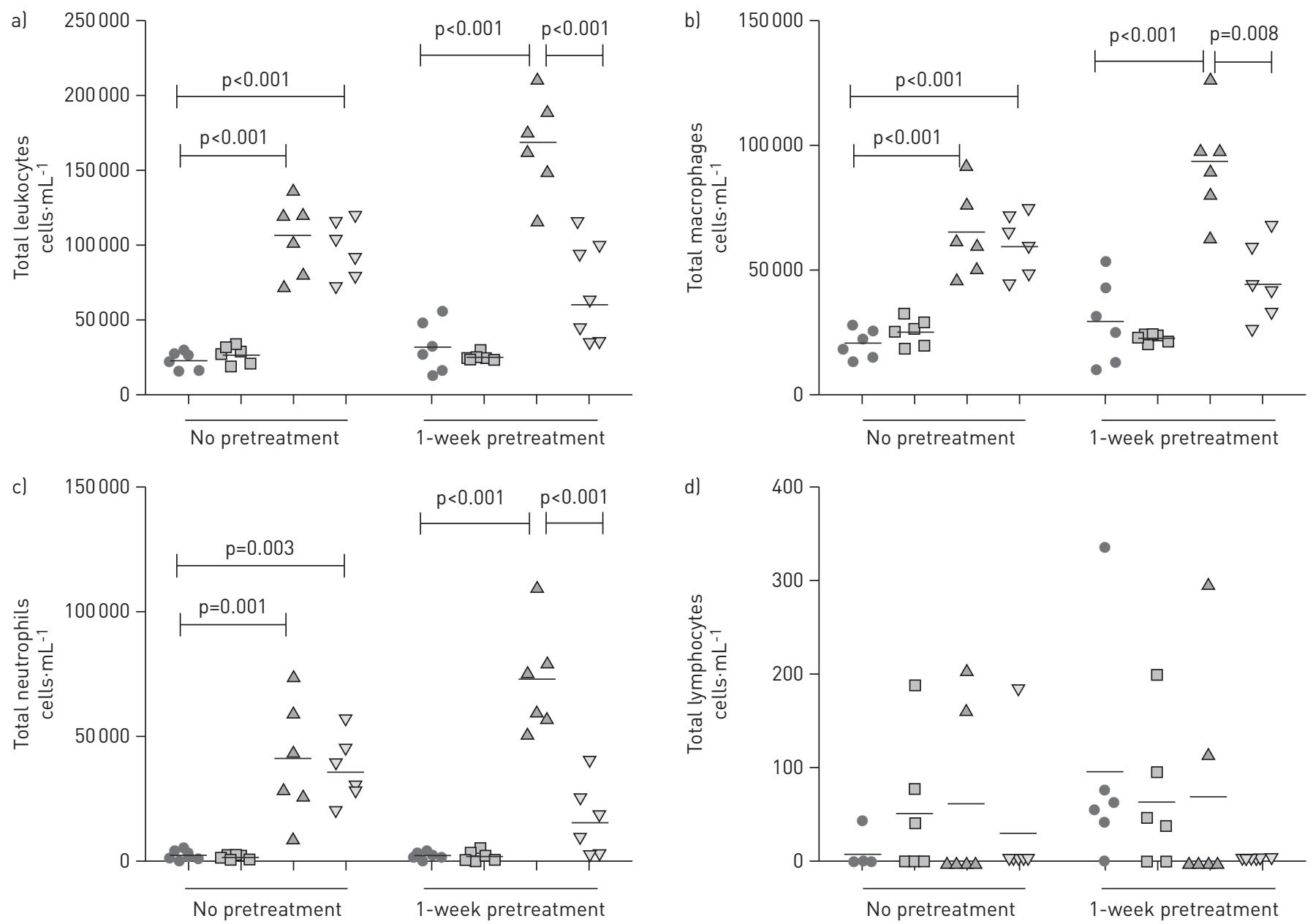

FIGURE 1 Pretreatment with simvastatin 1 week prior to tobacco smoke exposure, and continued throughout the exposure time-frame, reduced tobacco smokeinduced leukocyte recruitment to the lung, while treatment only on the smoke exposure days had no effect. a) Total leukocytes recovered in the bronchoalveolar lavage fluid (BALF). b) Total macrophages recovered in the BALF. c) Total neutrophils recovered in the BALF. d) Total lymphocytes recovered in the BALF. All groups were analysed by one-way ANOVA with Bonferroni corrections for multiple comparisons.

\section{Results}

Simvastatin requires a pretreatment period to reduce leukocyte recruitment to the lung

To determine whether simvastatin can prevent or reduce tobacco smoke-induced lung inflammation, we measured leukocytes recovered in the BALF. 3 days of cigarette smoke exposure significantly increased the total number of leukocytes, macrophages and neutrophils (fig. 1) in rats when compared with rats exposed to filtered air or filtered air plus simvastatin exposure. Lymphocytes made up $<3 \%$ of the total cells and were not increased by exposure to tobacco smoke. Spontaneously hypertensive rats treated with simvastatin for 1 week prior to smoke exposure and during the exposure course had reduced tobacco-smoke-induced total leukocytes, macrophages and neutrophils recruited to the lung compared with spontaneously hypertensive rats receiving a smoke-only treatment. By contrast, treatment with simvastatin concurrently with smoke exposure days had no effect, indicating that the anti-inflammatory effect of simvastatin is not immediate or a pre-exposure treatment period is required for the greatest benefit.

\section{Simvastatin prevents airway inflammation and epithelial injury}

To determine whether simvastatin can prevent or reduce tobacco smoke-induced damage to the lung, we evaluated haematoxylin and eosin stained lung sections. Airway epithelial injury was quantified by measuring the percentage of sloughed airway epithelium (fig. 2). Rats exposed only to tobacco smoke had extensive damage to the airways, including cellular infiltration into the subepithelial space and marked denudation of the epithelial layer. Numerous neutrophils and macrophages were also present in the airways. 

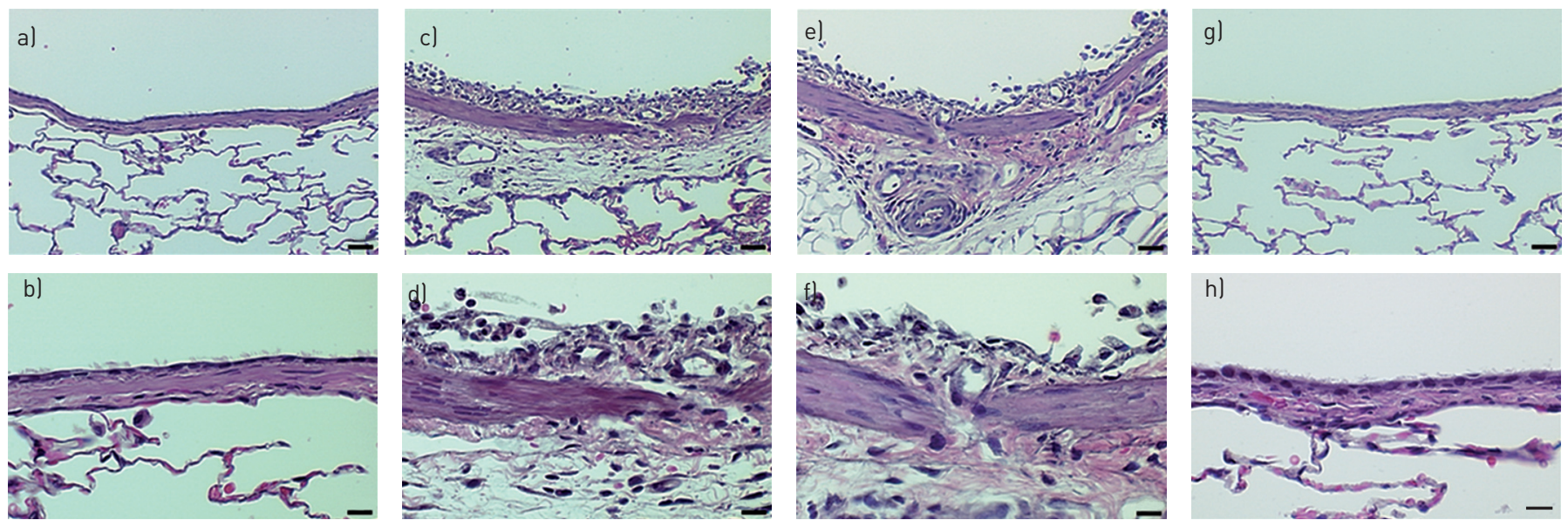
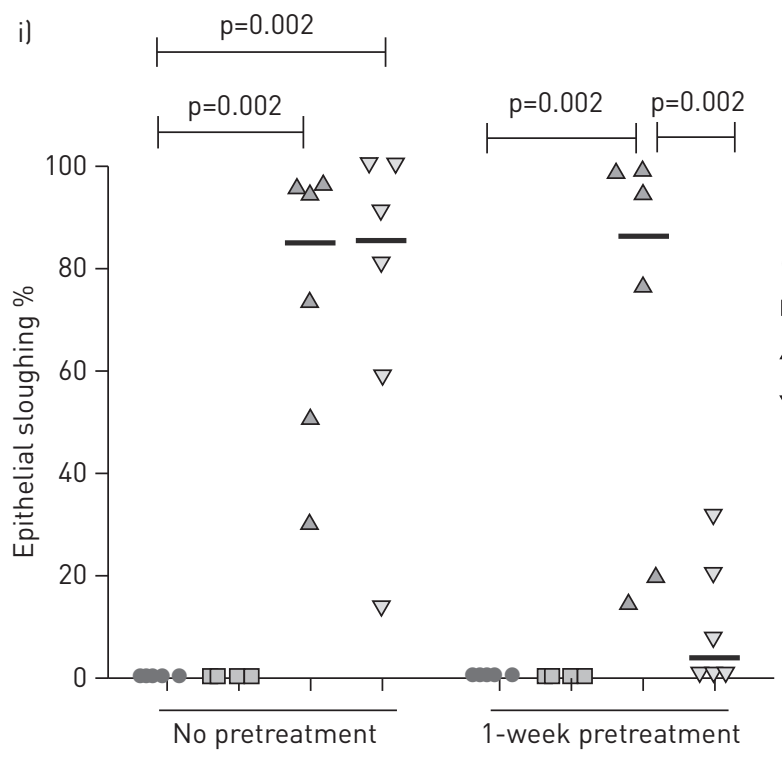

\section{- Air \\ $\square$ Air+simvastatin \\ $\triangle$ Smoke}

$\nabla$ Smoke+simvastatin

FIGURE 2 Epithelial and inflammatory changes to the bronchial wall of spontaneous hypertensive rats exposed to filtered air or tobacco smoke and treated with simvastatin. Exposure to only tobacco smoke was associated with significant airway epithelial injury and sloughing. Pretreatment with simvastatin provided almost complete epithelial protection to tobacco smoke if given 1 week prior to and during exposure to tobacco smoke, but not if treatment only began concurrently with exposure to tobacco smoke. Haematoxilin and eosin staining of bronchial airways at interpulmonary generation 2 in rats exposed to: a, b) filtered air; $c$, d) tobacco smoke; e, f) smoke plus simvastatin with no pretreatment; and g, h) smoke plus simvastatin with 1 week pretreatment with simvastatin. i) Quantitative analysis of the percentage of airway epithelial sloughing, data were analysed using the Wilcoxon rank sum test. a, c, e and g) scale bars $=25 \mu \mathrm{m}$. b, d, f and h) scale bars $=10 \mu \mathrm{m}$.

The lung histology of rats treated with simvastatin on smoke exposure days appeared identical to the histology from the tobacco smoke only group. However, rats pretreated with simvastatin for 1 week prior to smoke exposure (and that continued through the smoke exposure period) prevented tobacco-smokeinduced large airway epithelial injury $(\mathrm{p}=0.02)$ (fig. 2 ). Four of the six rats had virtually no damage or signs
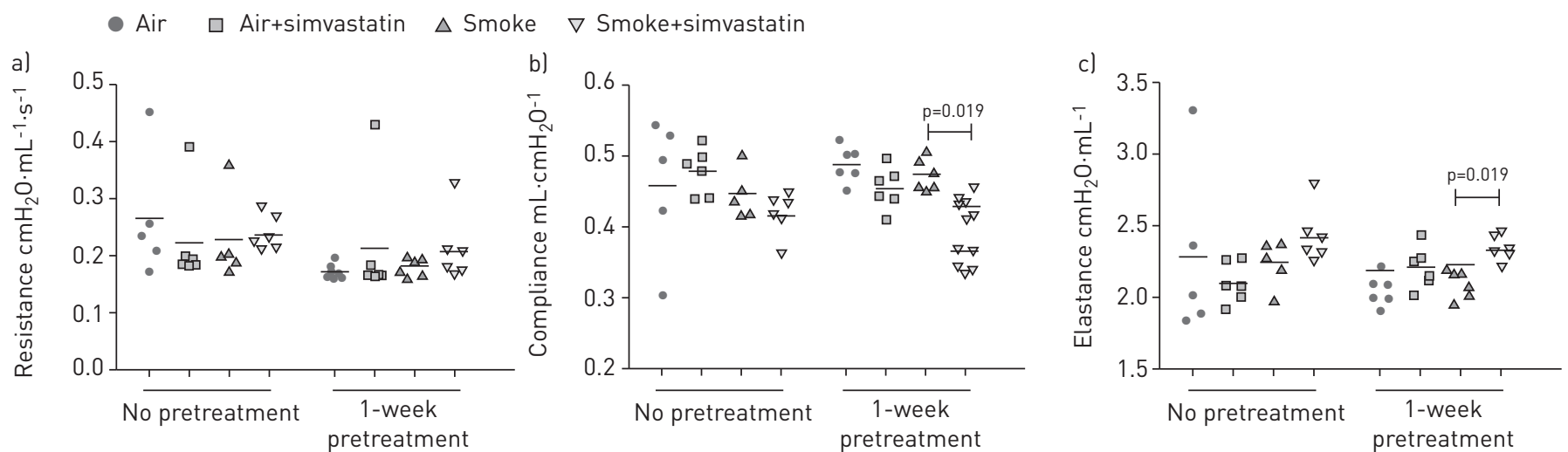

FIGURE 3 Treatment with simvastatin for 1 week prior to smoke exposure and continued during the smoke-exposure period reduced lung compliance and increased lung elastance, while simvastatin treatment only on exposure days had no effect. a) Resistance, b) compliance, c) elastance. All groups were analysed by one-way ANOVA with Bonferroni corrections for multiple comparisons. 
TABLE 1 Weight change over the 10 days of the study

$\begin{array}{lc}\text { No pretreatment } & \\ \text { Air } & 26 \pm 9 \\ \text { Air+simvastatin } & 23 \pm 7 \\ \text { Smoke } & -23 \pm 7 \\ \text { Smoke+simvastatin } & -33 \pm 11 \\ \text { 1-week pretreatment } & \\ \text { Air } & 12 \pm 10 \\ \text { Air+simvastatin } & -23 \pm 5 \\ \text { Smoke } & -28 \pm 7 \\ \text { Smoke+simvastatin } & -53 \pm 8\end{array}$

$\begin{array}{cc}1 & <0.001 \\ 1 & <0.001 \\ <0.001 & 1 \\ <0.001 & 0.476 \\ 1 & <0.001 \\ <0.001 & <0.001 \\ <0.001 & 1 \\ <0.001 & <0.001\end{array}$

Data are presented as mean \pm SD. Tobacco smoke or treatment with simvastatin for 1 week prior to smoke exposure and continued with exposure to tobacco smoke caused weight loss, while treatment with simvastatin only on the tobacco smoke exposure days had no effect. All groups were analysed by one-way ANOVA with Bonferroni corrections for multiple comparisons.

of inflammation and were indistinguishable from the filtered air controls. One rat had a small amount of cellular infiltration in the epithelium and a few inflammatory cells in the airway, and the other rat had clear damage and inflammation in the lung but to a lesser extent than all but one of the rats in the tobacco smoke only group.

\section{Simvastatin alters pulmonary function}

3 days of exposure to tobacco smoke did not significantly alter airway resistance, respiratory system compliance or elastance (fig. 3). However, simvastatin pretreatment reduced lung compliance (fig. 3b) and increased lung elastance (fig. 3c) when compared with the smoke only treatment. Simvastatin given only on exposure days did not alter pulmonary function.

\section{Simvastatin causes weight loss}

Rats only exposed to tobacco smoke or to tobacco smoke plus simvastatin, regardless of when simvastatin treatment began, had significant weight loss compared with corresponding filtered air exposed animals (table 1). Pretreatment with simvastatin was associated with a weight loss, whether the animals were

a)
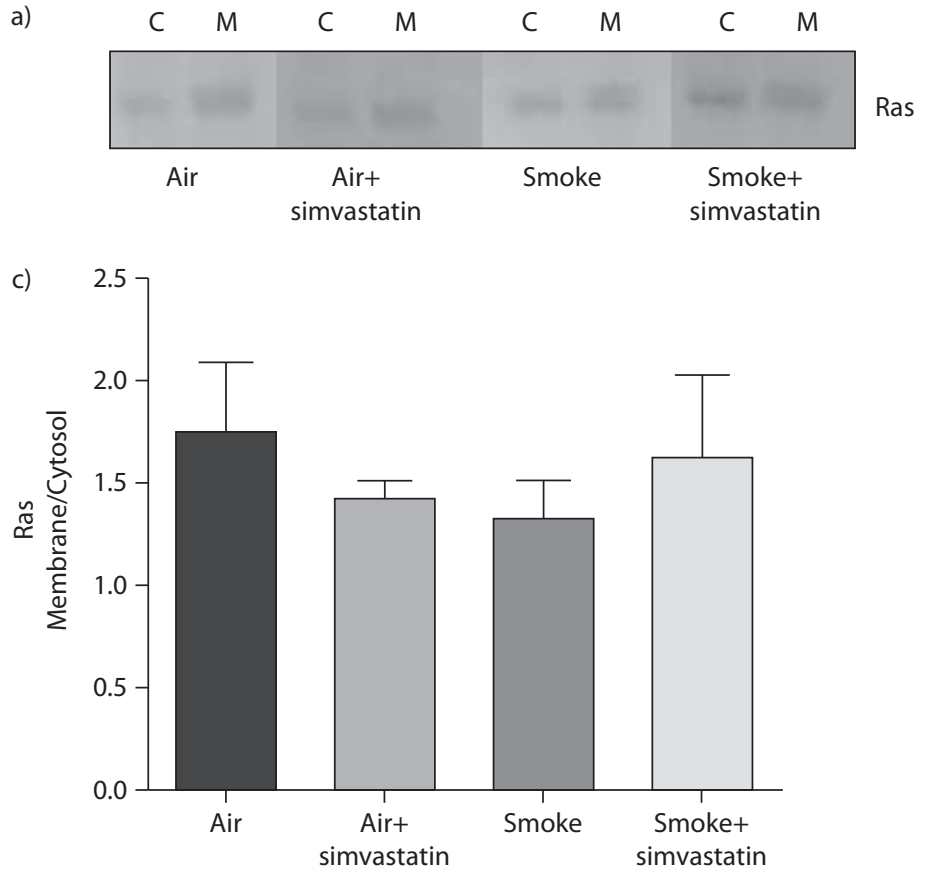

b)

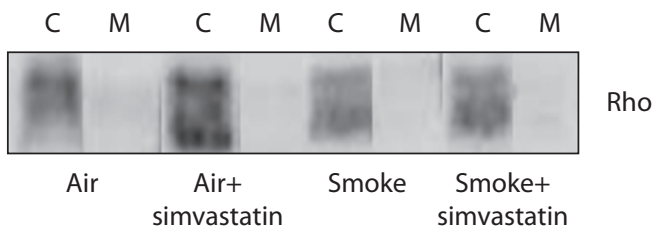

d)

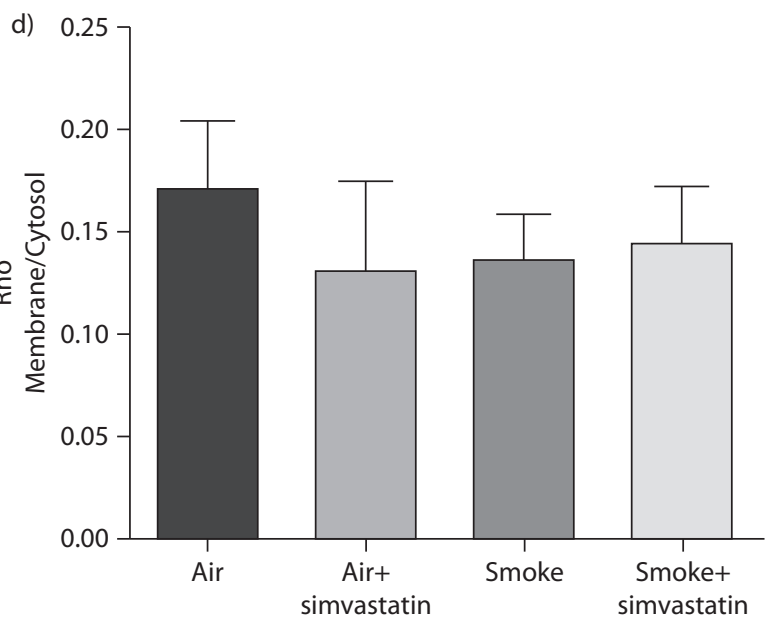

FIGURE 4 Treatment with simvastatin for 1 week prior to smoke exposure and continued during the exposure did not change localisation of Ras and Rho between the cytosolic (C) and membrane (M) fractions. a) Western blots of active Ras and c) membrane to cytosol ratio of Western blot densitometry. b) Western blots of active Rho and d) membrane to cytosol ratio of Western blot densitometry. All groups were analysed by one-way ANOVA. 
FIGURE 5 Treatment with simvastatin for 1 week prior to smoke exposure and continued during the exposure had no effect on total cholesterol in rat whole lung homogenate. Total cholesterol in whole lung homogenate lysates was measured and normalised to total protein, as described in the Materials and Methods. All groups were analysed by one-way ANOVA.

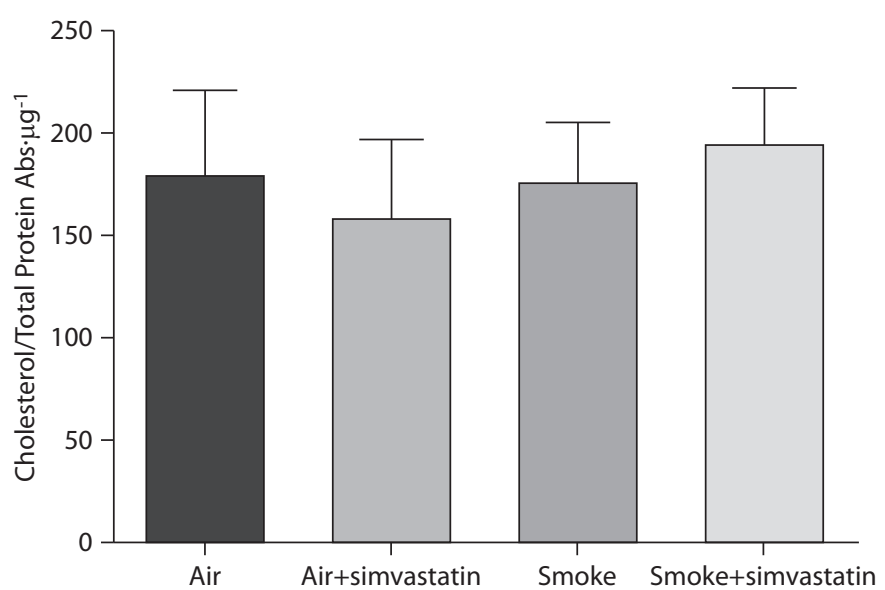

exposed to filtered air or tobacco smoke. In fact, animals exposed to tobacco smoke and pretreated with simvastatin lost more weight than any other group.

\section{Simvastatin did not alter lung Rho or Ras GTPase membrane localisation}

Part of the anti-inflammatory effect of statins has been proposed to occur by inhibition of GTPase membrane localisation $[10,19,24,25,40]$. Therefore, we tested whether simvastatin attenuated or inhibited tobacco smoke-induced membrane translocation of Ras and Rho. We did not detect smoke- or simvastatininduced changes in Ras or Rho translocation from the cytosol to the membrane in whole lung homogenate (fig. 4).

\section{Simvastatin did not alter whole lung cholesterol levels}

The mevalonate pathway branches downstream into the isoprenoids farnesyl pyrophosphate and geranylgeranyl pyrophosphate, and ultimately cholesterol. Since the statins deplete serum cholesterol as their main effect and given that cholesterol has been associated with increased lung inflammation in some animal models [41], we evaluated whole lung total cholesterol levels. We did not observe any smoke- or simvastatin-induced changes in lung total cholesterol in any of our experiments (fig. 5).

\section{Simvastatin reduces oxidative stress in smoke exposed rats}

We investigated the effect of simvastatin on oxidative stress as a possible mechanism of simvastatin's prevention of airway injury. HO-1 expression is increased with oxidative stress and it was assayed as a

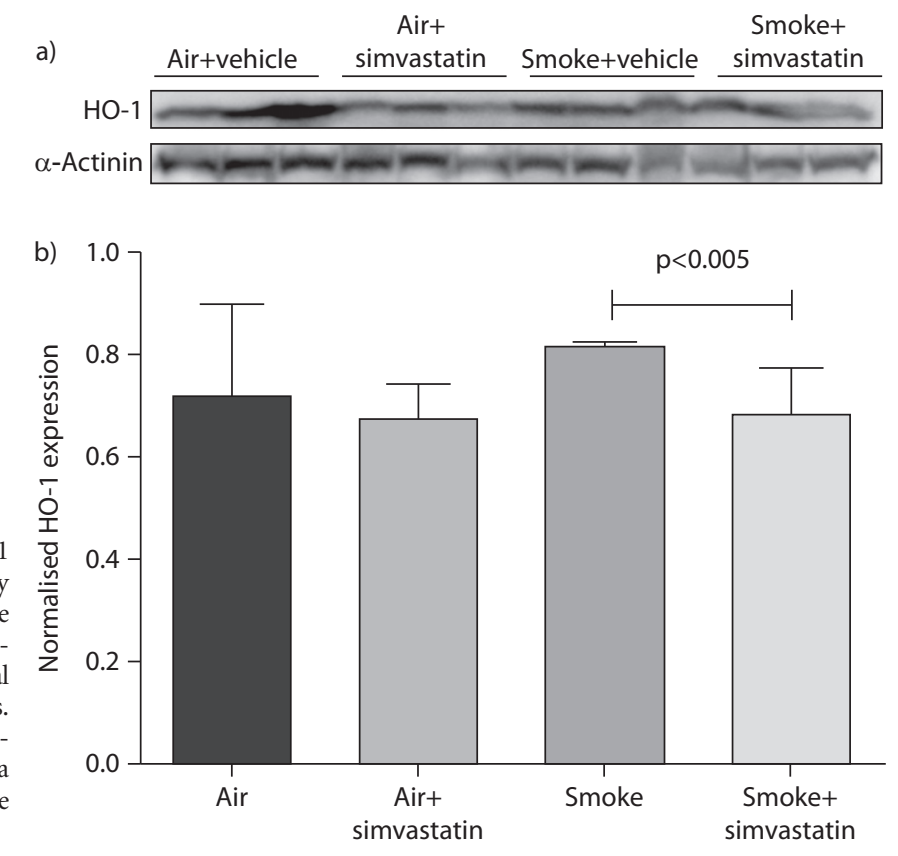

FIGURE 6 Haem oxygenase (HO)-1 protein expression was measured by Western blot as a marker for oxidative stress. a) Western blot of HO-1 and $\alpha$ actinin (as a control) from $100 \mu \mathrm{g}$ of total proteins from whole lung homogenates. b) Densitometric analysis of HO- 1 expression normalised to $\alpha$-actinin control. Data are presented as mean \pm SEM. p-value determined by Wilcoxon Rank sum test. 
marker of oxidative stress. In smoke exposed rats, treatment with simvastatin for 1 week prior to smoke exposure and during the exposure course significantly decreased HO-1 expression by Western blot (fig. 6). However, smoke-induced increases did not reach significance when compared with filtered air controls. Simvastatin has been reported to increase HO-1 [42-47], but it did not increase HO-1 expression in the rats exposed to filtered air, indicating that this effect did not occur in this study.

\section{Discussion}

This study demonstrates a causal relationship between statin use and the prevention of tobacco smokeinduced inflammatory cell recruitment to the lung and acute bronchial epithelial damage. We show three major novel findings regarding the protective anti-inflammatory effects of statins in smoke-induced lung damage: 1) pretreatment with simvastatin 1 week prior to tobacco smoke exposure not only reduces acute inflammatory cell recruitment to the lung, but also completely prevents smoke-induced acute airway epithelial injury and denudation; 2) the anti-inflammatory effect of simvastatin requires a 1-week pretreatment period, whereas there was no benefit when simvastatin was given concurrently with smoke exposure, indicating that the underlying mechanism of the anti-inflammatory effect of simvastatin is not immediate and must precede smoke exposure; and 3) these results were independent of simvastatin's effect on recovery, mucus accumulation and airway remodelling, changes that only occur with longer tobacco smoke exposures in our spontaneously hypertensive rat model.

The above findings suggest that statins may be appropriate for patients with a wide variety of bronchial diseases, such as the airways disease component of COPD (i.e. chronic bronchitis and not only emphysema). It is unclear why simvastatin protected the spontaneously hypertensive rats from acute airway epithelial damage. Systemic treatment with simvastatin may prevent smoke-induced damage via direct epithelial effects or prevent the secondary damage incurred by the epithelium from the influx of activated leukocytes. Other possibilities include statin antioxidative properties, which in the case of smoke-induced injury may play a major role [48-51]. We investigated whether treatment with simvastatin for 1 week prior to smoke exposure and during the exposure reduced HO-1, a marker of oxidative stress. Simvastatin significantly reduced HO-1 expression in smoke-exposed rats, supporting the hypothesis that simvastatin prevents airway injury by reducing oxidative stress. However, care should be taken not to over interpret these results because smoke induced increases in HO-1 expression compared to filtered air control did not reach significance. Thus, it is not clear how much oxidative stress contributed to airway injury in this study. Additionally, we do not know whether any oxidative stress is a direct result of smoke- exposure or secondary to neutrophil activation and subsequent respiratory burst.

Statin "preconditioning" appears to be a critical process for simvastatin's anti-inflammatory effect, which depended on the 1-week pretreatment period. We speculate that preconditioning could be due to the $a$ priori modulation of immune cell responses $[52,53]$, decreased pro-inflammatory cytokine/chemokine gene expression at the level of the airway epithelium $[54,55]$ or mesenchyme, or reduced vascular recruitment of leukocytes into lung tissue [56-58], amongst other mechanisms. Statins have been shown to inhibit lymphocyte function-associated antigen (LFA)-1 by binding to it and preventing high affinity binding of leukocytes to vascular endothelium to facilitate transmigration into inflamed tissues. We speculate that simvastatin is not preventing leukocyte recruitment through inhibition of LFA-1 because it would not require pretreatment.

Given the central role of the airway epithelium in both innate and adaptive immune responses [59, 60], where it is the central mediator of smoke-induced pro-inflammatory responses, additional studies are required to elucidate the protective effect of statins on the bronchial epithelium for statin dosing and administration. Future work is needed to determine whether simvastatin is acting on the lung tissue or directly on leukocytes or both to prevent leukocyte recruitment.

By demonstrating that simvastatin reduces both inflammatory cell recruitment to the lung and airway injury before the development of mucus accumulation and airway remodelling, we show that simvastatin prevents inflammatory cell recruitment and acute airway injury independently of these other important factors. Our results suggest that the short-term statin benefits in smoke-induced lung injury, which may be an early event in some phenotypes of COPD, do not depend on reducing mucus accumulation and airway remodelling. Within 10 days of treatment there is clear protection from statins in preventing further airway epithelial injury and leukocyte recruitment. The latter is a key component of acute COPD exacerbations and may, at least in part, explain why simvastatin use in large human epidemiological studies has been associated with a reduced risk of COPD exacerbations and improved lung function $[11,18]$.

Prior studies have shown that simvastatin can prevent or reverse smoke-induced emphysema and reverse pulmonary hypertension [29, 30], and when given chronically over 16 weeks with tobacco smoke, simvastatin attenuates smoke-induced small airway remodelling [31]. To our knowledge, our study is the 
first to report that systemic pretreatment with simvastatin inhibits smoke-induced bronchial epithelial injury and denudation. The beneficial effects of simvastatin in our model occurred within 10 days, which is a relatively short time period. Other animal studies have shown statins have an anti-inflammatory effect in the lung; however, the statin was administered during the animal's lung development and for a month (or longer) [21, 29, 31].

The anti-inflammatory effects of statins are thought to be mediated partly via inhibition of isoprenoid synthesis. Therefore, we investigated if simvastatin reduced the pool of available farnesyl pyrophosphate and geranylgeranyl pyrophosphate, thereby decreasing the proportion of isoprenylated or membrane-bound Ras and Rho, respectively. We expected to see smoke-induced cell membrane translocation (or activation) of Ras and/or Rho in our homogenised whole rat lungs. However, we did not detect smoke-induced or simvastatin-induced translocalisation of these GTPases (between the cytosolic and membrane fractions), indicating that any smoke-induced changes may be transient, subtle or restricted to certain cells and/or compartments within the lung, and not detectable in whole lung homogenate. Given these possibilities, testing whether co-treatment with geranylgeranyl pyrophosphate and farnesyl pyrophosphate, the isoprenoid molecules upstream of Rho and Ras activation, respectively, reverse the effects of simvastatin may be the best way to determine whether Rho and Ras activation modulates the protective effects of simvastatin.

Rats pretreated with simvastatin and exposed to tobacco smoke had reduced lung compliance (and increased lung elastance) (fig. 3). In humans, COPD typically manifests with abnormally high lung compliance due to emphysema and the resultant loss of lung elastic recoil. Thus, normalising or lowering lung compliance is usually desirable in COPD. However, the acute smoke exposure does not mimic this human pathophysiology. We speculate that the decrease in lung compliance may be due to a reduction in alveolar surfactant production [28], rather than due to the anti-inflammatory effect of simvastatin, because 3 days of smoke exposure did not alter compliance when compared with filtered-air controls. Also, any alterations in local cholesterol concentrations in resident alveolar cells due to simvastatin were not detectible by our assay; thus, we cannot relate this to cholesterol per se.

We were surprised to see a weight loss effect in the "pretreatment" experimental arm at the $20 \mathrm{mg} \cdot \mathrm{kg}^{-1}$ simvastatin dose because both the literature and our previous work did not reveal any evidence of toxicity or gross weight loss in both rats and mice at this dose or a higher dosage $[24,37]$. There is no analogy in humans. Statin use does not cause weight loss in humans based on clinical reports covering the past 30 years of continued statin use.

This work highlights simvastatin as a potential novel therapy for tobacco smoke-induced inflammatory lung diseases, such as COPD. We demonstrate that simvastatin attenuates acute tobacco smoke-induced leukocyte recruitment to the lung and further show that simvastatin pretreatment for 1 week almost completely prevents smoke-induced acute bronchial epithelial injury. The protective statin effect may be due to direct effects on the lung or mucosal surface during leukocyte-airway epithelial cell interactions [37, 61]. We envision simvastatin (and possibly other statins) as having the potential to become important adjunctive therapies in COPD, added to the current standard inhaler regimens. Additional animal studies are needed to investigate the underlying mechanisms of action. Also, ongoing clinical trials in both asthma and COPD will be of high interest.

\section{References}

$1 \quad$ Minai OA, Benditt J, Martinez FJ. Natural history of emphysema. Proc Am Thorac Soc 2008; 5: 468-474.

2 Snider GL. Epidemiology and natural history of COPD. In Leff AR, ed. Pulmonary and Critical Care Pharmacology and Therapeutics. New York, McGraw-Hill, 1996; pp. 821-828.

3 Zeki AA, Schivo M, Chan AL, et al. Geoepidemiology of COPD and idiopathic pulmonary fibrosis. J Autoimmun 2010; 34: J327-J338.

4 Jeffery PK. Structural and inflammatory changes in COPD: a comparison with asthma. Thorax 1998; 53: 129-136.

5 Fournier M, Lebargy F, Le Roy LF, et al. Intraepithelial T-lymphocyte subsets in the airways of normal subjects and of patients with chronic bronchitis. Am Rev Respir Dis 1989; 140: 737-742.

6 Saetta M, Turato G, Facchini FM, et al. Inflammatory cells in the bronchial glands of smokers with chronic bronchitis. Am J Respir Crit Care Med 1997; 156: 1633-1639.

7 Grashoff WF, Sont JK, Sterk PJ, et al. Chronic obstructive pulmonary disease: role of bronchiolar mast cells and macrophages. Am J Pathol 1997; 151: 1785-1790.

8 Saetta M, Di Stefano A, Turato G, et al. $\mathrm{CD}^{+}$T-lymphocytes in peripheral airways of smokers with chronic obstructive pulmonary disease. Am J Respir Crit Care Med 1998; 157: 822-826.

9 Zeki AA, Kenyon NJ, Goldkorn T. Statin drugs, metabolic pathways, and asthma: a therapeutic opportunity needing further research. Drug Metab Lett 2011; 5: 40-44.

10 Takeda N, Kondo M, Ito S, et al. Role of RhoA inactivation in reduced cell proliferation of human airway smooth muscle by simvastatin. Am J Respir Cell Mol Biol 2006; 35: 722-729.

11 Alexeeff SE, Litonjua AA, Sparrow D, et al. Statin use reduces decline in lung function: VA Normative Aging Study. Am J Respir Crit Care Med 2007; 176: 742-747. 
12 Hothersall E, McSharry C, Thomson NC. Potential therapeutic role for statins in respiratory disease. Thorax 2006; 61: 729-734.

13 Lawes CM, Thornley S, Young R, et al. Statin use in COPD patients is associated with a reduction in mortality: a national cohort study. Prim Care Respir J 2012; 21: 35-40.

14 Søyseth V, Brekke PH, Smith P, et al. Statin use is associated with reduced mortality in COPD. Eur Respir J 2007; 29: 279-283.

15 Frost FJ, Petersen $\mathrm{H}$, Tollestrup $\mathrm{K}$, et al. Influenza and COPD mortality protection as pleiotropic, dose-dependent effects of statins. Chest 2007; 131: 1006-1012.

16 Mancini GB, Etminan M, Zhang B, et al. Reduction of morbidity and mortality by statins, angiotensin-converting enzyme inhibitors, and angiotensin receptor blockers in patients with chronic obstructive pulmonary disease. J Am Coll Cardiol 2006; 47: 2554-2560.

17 Keddissi JI, Younis WG, Chbeir EA, et al. The use of statins and lung function in current and former smokers. Chest 2007; 132: 1764-1771.

18 Blamoun AI, Batty GN, DeBari VA, et al. Statins may reduce episodes of exacerbation and the requirement for intubation in patients with COPD: evidence from a retrospective cohort study. Int J Clin Pract 2008; 62: 1373-1378.

19 Maher BM, Dhonnchu TN, Burke JP, et al. Statins alter neutrophil migration by modulating cellular Rho activity-a potential mechanism for statins-mediated pleotropic effects? J Leukoc Biol 2009; 85: 186-193.

20 Sadeghi MM, Collinge M, Pardi R, et al. Simvastatin modulates cytokine-mediated endothelial cell adhesion molecule induction: involvement of an inhibitory G protein. J Immunol 2000; 165: 2712-2718.

21 Jacobson TA. Comparative pharmacokinetic interaction profiles of pravastatin, simvastatin, and atorvastatin when coadministered with cytochrome P450 inhibitors. Am J Cardiol 2004; 94: 1140-1146.

22 Sun XF, Wang LL, Wang JK, et al. Effects of simvastatin on lung injury induced by ischaemia-reperfusion of the hind limbs in rats. J Int Med Res 2007; 35: 523-533.

23 Fessler MB, Young SK, Jeyaseelan S, et al. A role for hydroxy-methylglutaryl coenzyme a reductase in pulmonary inflammation and host defense. Am J Respir Crit Care Med 2005; 171: 606-615.

24 Zeki AA, Franzi L, Last J, et al. Simvastatin inhibits airway hyperreactivity: implications for the mevalonate pathway and beyond. Am J Respir Crit Care Med 2009; 180: 731-740.

25 Liao JK. Isoprenoids as mediators of the biological effects of statins. J Clin Invest 2002; 110: 285-288.

26 Lee JH, Lee DS, Kim EK, et al. Simvastatin inhibits cigarette smoking-induced emphysema and pulmonary hypertension in rat lungs. Am J Respir Crit Care Med 2005; 172: 987-993.

27 Ou XM, Wen FQ, Uhal BD, et al. Simvastatin attenuates experimental small airway remodelling in rats. Respirology 2009; 14: 734-745.

28 Davis BB, Shen Y, Tancredi DJ, et al. Leukocytes are recruited through the bronchial circulation to the lung in a spontaneously hypertensive rat model of COPD. PLoS One 2012; 7: e33304.

29 Davis BB, Liu JY, Tancredi DJ, et al. The anti-inflammatory effects of soluble epoxide hydrolase inhibitors are independent of leukocyte recruitment. Biochem Biophys Res Commun 2011; 410: 494-500.

$30 \mathrm{Yu}$ B, Kodavanti UP, Takeuchi M, et al. Acute tobacco smoke-induced airways inflammation in spontaneously hypertensive rats. Inhal Toxicol 2008; 20: 623-633.

31 Zhong CY, Zhou YM, Pinkerton KE. NF- $\mathrm{KB}$ inhibition is involved in tobacco smoke-induced apoptosis in the lungs of rats. Toxicol Appl Pharmacol 2008; 230: 150-158.

32 Zhong CY, Zhou YM, Douglas GC, et al. MAPK/AP-1 signal pathway in tobacco smoke-induced cell proliferation and squamous metaplasia in the lungs of rats. Carcinogenesis 2005; 26: 2187-2195.

33 Smith KR, Pinkerton KE, Watanabe T, et al. Attenuation of tobacco smoke-induced lung inflammation by treatment with a soluble epoxide hydrolase inhibitor. Proc Natl Acad Sci USA 2005; 102: 2186-2191.

34 Smith KR, Uyeminami DL, Kodavanti UP, et al. Inhibition of tobacco smoke-induced lung inflammation by a catalytic antioxidant. Free Radic Biol Med 2002; 33: 1106-1114.

35 Teague SV, Pinkerton KE, Goldsmith M, et al. Sidestream cigarette smoke generation and exposure system for environmental tobacco smoke studies. Inhal Toxicol 1994; 6: 79-93.

36 Pillsbury HC Jr. Review of the Federal Trade Commission method for determining cigarette tar and nicotine yield. Smoking and Tobacco Control Monograph No. 7; pp. 9-14. Available from: http://cancercontrol.cancer.gov/brp/ tcrb/monographs/7/m7_2.pdf.

37 Chen YJ, Chen P, Wang HX, et al. Simvastatin attenuates acrolein-induced mucin production in rats: involvement of the Ras/extracellular signal-regulated kinase pathway. Int Immunopharmacol 2010; 10: 685-693.

38 Vickers S, Duncan CA, Chen IW, et al. Metabolic disposition studies on simvastatin, a cholesterol-lowering prodrug. Drug Metab Dispos 1990; 18: 138-145.

39 Bratt JM, Franzi LM, Linderholm AL, et al. Arginase enzymes in isolated airways from normal and nitric oxide synthase 2-knockout mice exposed to ovalbumin. Toxicol Appl Pharmacol 2009; 234: 273-280.

40 Eccles KA, Sowden H, Porter KE, et al. Simvastatin alters human endothelial cell adhesion molecule expression and inhibits leukocyte adhesion under flow. Atherosclerosis 2008; 200: 69-79.

41 Yeh YF, Huang SL. Enhancing effect of dietary cholesterol and inhibitory effect of pravastatin on allergic pulmonary inflammation. J Biomed Sci 2004; 11: 599-606.

42 Mrad MF, Mouawad CA, Al-Hariri M, et al. Statins modulate transcriptional activity of heme-oxygenase-1 promoter in NIH 3T3 cells. J Cell Biochem 2012; 113: 3466-3475.

43 Tu YP, Chuang SJ, Chen SC, et al. Simvastatin induces the expression of hemeoxygenase-1 against ischemiareperfusion injury on the testes in rats. Toxicol Lett 2011; 207: 242-250.

44 Hsieh $\mathrm{CH}$, Sun CK, Lu TH, et al. Simvastatin induces heme oxygenase-1 expression but fails to reduce inflammation in the capsule surrounding a silicone shell implant in rats. J Surg Res 2011; 168: 272-280.

45 Hinkelmann U, Grosser N, Erdmann K, et al. Simvastatin-dependent up-regulation of heme oxygenase-1 via mRNA stabilization in human endothelial cells. Eur J Pharm Sci 2010; 41: 118-124.

46 Min KS, Lee YM, Hong SO, et al. Simvastatin promotes odontoblastic differentiation and expression of angiogenic factors via heme oxygenase-1 in primary cultured human dental pulp cells. J Endod 2010; 36: 447-452.

47 Hsu HH, Ko WJ, Hsu JY, et al. Simvastatin ameliorates established pulmonary hypertension through a heme oxygenase-1 dependent pathway in rats. Respir Res 2009; 10: 32. 

microdomains and oxidative stress. Circulation 2004; 109: II34-II41.

49 Bandoh T, Sato EF, Mitani H, et al. Antioxidative potential of fluvastatin via the inhibition of nicotinamide adenine dinucleotide phosphate (NADPH) oxidase activity. Biol Pharm Bull 2003; 26: 818-822.

50 Altintas ND, Atilla $\mathrm{P}$, Iskit $\mathrm{AB}$, et al. Long-term simvastatin attenuates lung injury and oxidative stress in murine acute lung injury models induced by oleic acid and endotoxin. Respir Care 2011; 56: 1156-1163.

51 Guasti L, Marino F, Cosentino M, et al. Prolonged statin-associated reduction in neutrophil reactive oxygen species and angiotensin II type 1 receptor expression: 1-year follow-up. Eur Heart J 2008; 29: 1118-1126.

52 Greenwood J, Steinman L, Zamvil SS. Statin therapy and autoimmune disease: from protein prenylation to immunomodulation. Nat Rev Immunol 2006; 6: 358-370.

53 Ho PC, Chang KC, Chuang YS, et al. Cholesterol regulation of receptor-interacting protein 140 via microRNA-33 in inflammatory cytokine production. FASEB J 2011; 25: 1758-1766.

54 Murphy DM, Forrest IA, Corris PA, et al. Simvastatin attenuates release of neutrophilic and remodeling factors from primary bronchial epithelial cells derived from stable lung transplant recipients. Am J Physiol Lung Cell Mol Physiol 2008; 294: L592-L599.

55 Wang W, Le W, Ahuja R, et al. Inhibition of inflammatory mediators: role of statins in airway inflammation. Otolaryngol Head Neck Surg 2011; 144: 982-987.

56 Kinsella A, Raza A, Kennedy S, et al. The impact of high-dose statin therapy on transendothelial neutrophil migration and serum cholesterol levels in healthy male volunteers. Eur J Clin Pharmacol 2011; 67: 1103-1108.

57 Dunzendorfer S, Rothbucher D, Schratzberger P, et al. Mevalonate-dependent inhibition of transendothelial migration and chemotaxis of human peripheral blood neutrophils by pravastatin. Circ Res 1997; 81: 963-969.

58 Zhang S, Rahman M, Zhang S, et al. Simvastatin regulates CXC chemokine formation in streptococcal M1 proteininduced neutrophil infiltration in the lung. Am J Physiol Lung Cell Mol Physiol 2011; 300: L930-L939.

59 Parker D, Prince A. Innate immunity in the respiratory epithelium. Am J Respir Cell Mol Biol 2011; 45: 189-201.

60 Schleimer RP, Kato A, Peters A, et al. Epithelium, inflammation, and immunity in the upper airways of humans: studies in chronic rhinosinusitis. Proc Am Thorac Soc 2009; 6: 288-294.

61 Planaguma A, Pfeffer MA, Rubin G, et al. Lovastatin decreases acute mucosal inflammation via 15-epi-lipoxin A4. Mucosal Immunol 2010; 3: 270-279. 